\title{
Care in the time of coronavirus: Ethical considerations in head and neck oncology
}

\author{
Eli A. Gordin, MD, FACS ${ }^{1}$ \\ ${ }^{1}$ University of Texas - Southwestern Medical Center
}

May 5, 2020

\begin{abstract}
As COVID-19 continues to challenge the practice of head and neck oncology, clinicians are forced to make new decisions in the setting of the pandemic that impact the safety of their patients, their institutions and themselves. The difficulty inherent in these decisions is compounded by potentially serious ramifications to the welfare of patients and healthcare staff, amid a scarcity of data on which to base informed choices. This paper explores the risks of COVID-19 incurred while striving to uphold the standard of care in head and neck oncology. The ethical problems are assessed from the perspective of the cancer patient, healthcare provider, and other patients within the healthcare system. While no single management algorithm for head and neck cancer can be universally implemented, a detailed examination of these issues is necessary to formulate ethically sound treatment strategies.
\end{abstract}

1. Eli A. Gordin, MD, FACS

Assistant Professor: Otolaryngology - Head and Neck Surgery

University of Texas - Southwestern Medical Center

2. Andrew Day, MD, MPH

Assistant Professor: Otolaryngology - Head and Neck Surgery

University of Texas - Southwestern Medical Center

Dallas, TX

3. Lenka Stankova, MD

Assistant Professor: Otolaryngology - Head and Neck Surgery

University of Texas - Southwestern Medical Center

VA North Texas Health Care System

Dallas, TX

4. Elizabeth Heitman, Ph.D.

Professor: Psychiatry, Division of Ethics in Science and Medicine

University of Texas - Southwestern Medical Center

Dallas, TX

5. John Sadler, MD 
Professor: Psychiatry, Division of Ethics in Science and Medicine

University of Texas - Southwestern Medical Center

Dallas, TX

Grant support: none

Meeting presentations: none

Corresponding Author:

Eli A. Gordin, MD, FACS

Assistant Professor: Otolaryngology - Head and Neck Surgery

University of Texas - Southwestern Medical Center

2001 Inwood Rd

Dallas, TX 75390

Eli.gordin@utsouthwestern.edu

215-805-4354

Running title: Care in the time of coronavirus

Keywords: COVID-19, head and neck, oncology, ethics, cancer

Abstract:

As COVID-19 continues to challenge the practice of head and neck oncology, clinicians are forced to make new decisions in the setting of the pandemic that impact the safety of their patients, their institutions and themselves. The difficulty inherent in these decisions is compounded by potentially serious ramifications to the welfare of patients and healthcare staff, amid a scarcity of data on which to base informed choices.

This paper explores the risks of COVID-19 incurred while striving to uphold the standard of care in head and neck oncology. The ethical problems are assessed from the perspective of the cancer patient, healthcare provider, and other patients within the healthcare system. While no single management algorithm for head and neck cancer can be universally implemented, a detailed examination of these issues is necessary to formulate ethically sound treatment strategies.

Introduction:

The COVID-19 pandemic has significantly altered the daily practice of head and neck oncology throughout the world. Most head and neck cancers require urgent management within weeks, as extended delays may result in additional morbidity or even death. However, oncologic care has been critically challenged by pandemic-driven uncertainty and numerous, novel, multilevel COVID-19-specific risks. Now, in addition to treating patients' cancers, head and neck oncologists must also:

1. Protect the patient from infection with SARS-CoV-2 as well as minimizing risk factors for COVID19-related fatality in the event of infection

2. Prevent the spread of COVID-19 within healthcare institutions and the community

3. Minimize the risk of COVID-19 transmission to healthcare workers

4. Fairly allocate resources to all patients in a time of scarcity

What follows is an examination of the ethical implications involved in navigating the above risks. These issues need to be reconciled with the desire to deliver standard of care therapy amid great uncertainty and 
in the context of a shortage of resources. The hope is that, by describing these conflicts, clinicians will be better able to understand their own ethical perspectives and those of their colleagues while approaching treatment decisions.

\section{Uncertainty}

The lack of data concerning COVID-19 is immediately apparent to clinicians, especially as it relates to head and neck oncology. This new, widespread disease creates a high level of uncertainty which permeates further discussions. New studies are published daily. Estimates of the prevalence of COVID-19 vary widely. COVID-19 is not distributed uniformly throughout the world or even within the United States and guidelines are not necessarily generalizable to all institutions.

The rate of nosocomial transmission is not known. Exposure risk to healthcare workers is unclear, especially in the context of procedures that are generally necessary while delivering head and neck cancer care. The actual risk of contracting COVID-19 in the community or the healthcare setting cannot be specified.

Moreover, the wide variation in the presentation of COVID-19 makes it highly challenging for hospitals and clinicians to plan for the future care needs of their patients. SARS-CoV-2 infection may be unrecognizable, with minimal or no symptoms in some patients, while others may suffer catastrophic multi-organ system failure. This wide range of disease severity compounds the difficulty in predicting which clinical services will be overwhelmed as the pandemic evolves and, therefore, what resultant opportunity cost may be lost in diverting resources from head and neck patients to a projected crisis.

\section{Goals of care}

In an ideal setting, head and neck cancers are diagnosed, worked-up, and brought to treatment expediently, within a matter of days to weeks. Whether surgical resection, radiation, chemotherapy, immunotherapy, or a combination of modalities is employed should depend only on which treatment strategy gives the patient the greatest chance of actualizing his or her goals of care. Whether treatment is curative or palliative, organ preserving or excisional, standard or experimental, or whether treatment is completed in one sitting or over the course of many weeks should only depend on what is in the best interest of the patient - as expressed by that patient. Treatment should be guided by a multidisciplinary treatment team of healthcare professionals who provide the counseling and education regarding all possible treatment options that have been vetted with scientifically rigorous evidence.

The treatment decision is a personal one between a patient and his or her doctors and should be free, ideally, from external pressures such as finances, access, scheduling, and availability of resources. The physician should have only the interests of that one patient in mind and the patient should be able to focus on only those considerations that relate to adherence to the treatment plan, to the exclusion of other concerns.

These ideals are based on the four pillars of medical ethics: beneficence, nonmaleficence, autonomy, and justice, all of which are patient-centered. That is, we should do good to and for our patients, while avoiding doing harm, and allow the patient's needs and values to guide the treatment plan - as we would for any other patient in a similar situation. Although foundational, a standard of medical practice based on these principles alone would be poorly generalizable and neglects the practical limitations of inconvenient clinical situations and imperfect systems of care. It views the patient in isolation from his or her community and excludes the finite economics and resources of medicine and the associated implications to public health. It does not address the requirement that healthcare workers prevent harm to themselves, which in turn enables them to continue to render care. Finally, it does not recognize that the patient is motivated, in part, by practical responsibilities - financial, social and otherwise - that may serve as obstacles to treatment.

Medicine is not practiced in a vacuum, independent from society and politics. There are other factors that influence medical decision-making, at every level, that transgress the ideal framework of the textbook doctor-patient relationship. This was the case long before COVID-19 and will be the case far after. That is not to say that we were not practicing good medicine prior to COVID and it does not mean that we cannot continue to do so in the face of such a pandemic. However, as we invoke ethical principles in the name 
of quality medical care, it must be recognized that we are not above the influence of the practical, societal forces at play that have always been involved in the calculus of medical decision-making.

\section{Standards of Care}

Head and neck cancer treatment plans are generally formulated in adherence with the National Comprehensive Cancer Network (NCCN) guidelines. According to the NCCN "NCCN Guidelines are the recognized standard for clinical direction and policy in cancer care and are the most thorough and frequently updated clinical practice guidelines available in any area of medicine" Additionally, "The NCCN Guidelines provide recommendations based on the best evidence available at the time they are derived." (Emphasis added) [i]

Obviously the NCCN guidelines were derived before the novel coronavirus infected its first victim. Insufficient time has passed to amass necessary data regarding the risks of COVID-19 to both head and neck cancer patients and their providers. Therefore, we cannot know whether the current NCCN guidelines would stand as they are now if such risks were fully elucidated.

Certainly, the need to balance risk in the formulation of a treatment plan is not novel, and treatment guidelines are dictated by known risks. The risks of treatment failure are balanced against the risks of morbidity and mortality from proposed interventions. These concepts are incorporated into medical jargon. For example, the term "unresectable" is sometimes substituted as shorthand for stating that a surgical procedure involves a degree of risk that is deemed unacceptable relative to the perceived benefit of attempting surgical extirpation.

Currently, many organizations, including the NCCN, have advocated for practices that are not considered standard of care, in light of the COVID-19 pandemic. These include postponing office visits and radiology appointments for cancer surveillance, converting clinic visits to tele-health encounters when possible, and minimizing aerosol-generating procedures, such as flexible laryngoscopy.[ii]

As per the NCCN website's reference to COVID-19: "Individual clinical judgment is necessary; these recommendations cannot provide absolutes for alternate strategies during the COVID-19 outbreak." "Furthermore, some of these recommendations would not normally be considered standard of care or optimal but are reasonable under these unusual circumstances in which minimizing visits and potential exposure has become a priority." [iii]

This suggests that managing the perceived (though unknown) risk of spreading COVID-19 has superseded the standard of care in some situations, such as the follow up of post-treatment head and neck cancer patients. While these situations may not have a clear impact on the immediate management of the patient, it is possible that early detection of cancer recurrence could be missed because of such practice modifications. Whether this risk is justified by a reduction in the spread of COVID-19 to the patient, associated healthcare staff, and the population in general is not known.

\section{The patient's risk}

Available data shows that the chances of fatality from COVID-19 are significantly higher in patients with certain co-morbidities, including pulmonary disease, cancer, and immunocompromised status.[iv] [v] These conditions are common in head and neck cancer patients. It also appears that patients who are infected with SARS-CoV-2 who undergo major surgery have a significantly increased risk of mortality.[vi] Furthermore, because no effective treatments for COVID-19 are available at this time, the most effective management strategy is disease prevention.

The actual risks of surgery in the current context are unknown, and the information necessary to adequately counsel patients is not available. The 30-day mortality risk incurred by undergoing major head and neck surgery may be around $3 \%$ at baseline.[vii] [viii] If the patient's risk of mortality when undergoing major head and neck surgery during the COVID-19 pandemic were increased by a defined percentage, we could easily explain this to the patient. A surgical standard of care would prevail if the average overall survival of the surgical patient still exceeded that with non-surgical treatment. If the surgical patient's chance of 
mortality were increased beyond that of non-standard alternative therapy, the surgical standard would be inappropriate.

At this moment in time, it may be reasonable to assume that most patients who have head and neck cancer do not have COVID-19 and that they are more likely to die from that cancer, if left untreated, than they are from the infection. In cases where the efficacy of non-surgical treatment is inferior or unknown relative to a surgical standard of care, a compelling reason to forego safety for alternative therapy would be needed in this situation. When only patient benefit is considered, deviation from the standard of care for a known cancer becomes more difficult.

Deviation from the established standard of care would be more acceptable if there were a clear and present benefit to the patient. For example, if an institution was experiencing a major nosocomial outbreak of COVID-19, it might be prudent to avoid surgical therapy that would necessitate admission in favor of nonstandard treatment, thereby avoiding a delay in some form of care. Similarly, an institution without available ventilators or operating rooms due to a massive surge in COVID-19 would present the same problem. These examples assume that there are no other institutions where the patient could receive treatment. Given the constraints in health insurance and financial resources for many patients, this situation is certainly possible.

Any treatment offered to the patient requires detailed informed consent. For surgical excision, the added risk of mortality due to COVID-19 would ideally be described to the patient. If treatment is non-standard, this clearly must be articulated to the patient as well. We presently have insufficient information to counsel the patient in our normal capacity. Unfortunately, we must still explain these unknowns to the best of our ability.

\section{The provider's risk}

The appropriate PPE for mucosal head and neck cases in the setting of COVID-19 is in question. Aerosolgenerating procedures create an increased risk of respiratory viral infection to healthcare workers relative to other patient encounters. $[\mathrm{ix}][\mathrm{x}]$ The available data mainly concerns intubation and it is unclear what PPE is necessary to prevent SARS-CoV-2 transmission in surgeries where aerosolization is occurring, potentially for hours on end, far in excess of an intubation. This duration of continuous exposure would likely carry a much higher viral load and a greater severity of disease. Again, the exact risks are currently unknown.

The likelihood of operating on an unrecognized COVID-19 positive patient may be reduced by pre-operative testing, but considering the oft-quoted false negative rate of $20 \%$ for nasopharyngeal sampling for SARS-CoV2[xi], as well as the significant proportion of asymptomatic, infected individuals, the risk cannot be eliminated. Even for patients presenting as COVID-19 negative, the risk for community infection is continuous. Selfquarantine for preoperative patients, up to the time of surgery, would serve to further decrease this risk. Though the exact risk of transmission to the healthcare worker is unknown, statistical principles dictate that staff who engage in greater numbers of these cases would be placed at increased danger, especially as the viral cases proliferate in the community.

As risk increases, there comes a point at which surgeons may become uncomfortable with continuing their work. If the surgeon's risk were known, how might this information be incorporated into the formulation of the standard of care? Add to this the combined risk to all the healthcare workers involved, both during the procedure and in the post-operative setting, where aerosol-generating procedures continue to be necessary in caring for wounds and tracheostomies.

It could be argued that institutions now bear a burden to inform their staff of these exposure risks to SARSCoV-2. This communication could be implemented, in part, by incorporating review of any potential for transmission of infectious disease and specifically COVID-19 into the pre-surgical "timeout". This would at least assist in communicating a heightened risk to operating room personnel. As with patients, informing healthcare staff of the risks involved would respect the autonomy of those individuals, and allow each member of the treatment team to consent to participate willfully, utilizing safety precautions with which each provider is comfortable. As with the limits to informed consent of the patient, such disclosures are difficult because 
the risks in question are not yet quantifiable.

Healthcare workers have always faced some personal risk in the treatment of head and neck cancer. Radiation therapy and medical oncology personnel take precautions to prevent exposures to ionizing radiation and chemotherapeutic agents. In surgery, the risk of transmission of human immunodeficiency virus (HIV) and hepatitis-C virus (HCV) were present before the COVID-19 pandemic. Unlike the current pandemic, the above risks are now well defined and there is institutional policy and governmental regulation set in place to protect workers from them.

Relative to blood-borne disease, what constitutes an exposure event related to SARS-CoV-2 is unclear. A COVID-19 exposure from a patient who unknowingly harbored the virus would easily go unrecognized unless and until the healthcare worker or patient developed symptoms. Additionally, as opposed to HIV and HCV, there currently are no established medications to lessen the impact of an exposure to SARS-CoV-2.

For a time, lack of general knowledge regarding the transmission and infectivity of HIV fueled fears among healthcare workers. The situation with COVID-19 parallels the early days of HIV and we do not yet know whether our current infection control practices are too stringent or whether we should be increasing our usage of PPE and other measures. Time will certainly tell. While it has become clear that effective infection control practices for HIV are relatively simple precautions against blood-borne transmission, involving gloves, a surgical mask, and eye protection, it does not necessarily follow that this will be true of SARS-CoV-2. It may be prudent to err on the side of caution. This would maximize our adherence to the principle of nonmaleficence.

\section{A duty to treat?}

A complete discussion of a healthcare worker's duty to treat in the setting of his or her own personal risk is beyond the scope of this paper. The reader is referred to the work of Heidi Malm and her co-authors in 2008. ${ }^{12}$ Suffice it to say that, even amongst healthcare workers, there is no consensus regarding this topic. Modern medicine comprises so many different specialties and vocational roles that the heterogeneity of individuals within healthcare prevents a blanket statement of the degree to which personnel understand, expect, and consent to all possible occupational risk. Furthermore, any perceived burden of reciprocating prior benefits bestowed by society to the healthcare worker is not generalizable nor agreed upon. Lastly, only a few professional associations suggest that a duty to treat exits in the face of personal harm, with fewer incorporating it into an oath or pledge.[xii]

As for the physician treating head and neck cancer, it is not reasonable to suggest that subjecting oneself to physical harm while carrying out routine functions of patient care was somehow consciously or implicitly consented to by choosing this specialty, or that the physician is ethically obligated to work without regard for his or her wellbeing. Head and neck oncology physicians expect to work long hours and to make difficult choices while engaging in emotionally laden human interaction. They can expect to sacrifice time they might have spent with their families and their other passions for the responsibility of treating others' life-threatening cancers, but not their own health or that of their families.

Each practitioner must be allowed to decide the degree of risk he or she is willing to accept. Like all patients who contract COVID-19, elderly healthcare workers and those with medical co-morbidities are at increased risk for mortality and their exposure risk should be reduced as much as possible. This reality increases the need for clarity around what constitutes adequate respiratory PPE for aerosol-generating procedures, especially as maximal PPE may not be available for all providers. Without the support of good data to understand COVID-19 transmission and severity, institutions that do not make every effort to provide the highest level of PPE to staff participating in long duration, major, mucosal head and neck surgery expose these valuable healthcare professionals to unknown and potentially unacceptable risks. Forcing healthcare workers to accept these risks by threatening to withhold pay, revoke privileges, or other disciplinary action is coercive and unethical. Neglecting healthcare workers' welfare will only serve to compound the tragedy of the COVID-19 pandemic. 


\section{Allocation of resources}

The COVID-19 pandemic will tax the availability of medical resources in many areas where scarcity is not already a reality. In some locations both regular hospital and ICU beds are already in short supply, as well as ventilators. This shortage must be reconciled with the need to treat head and neck cancer patients who would best be addressed surgically. Additionally, head and neck operations require the expenditure of scarce PPE, and in many cases may necessitate enhanced PPE that are rarer still. The availability of such PPE is already problematic.

There may not be one universally accepted definition of rationing as it applies to healthcare. For many the term possesses pejorative undertones and harkens back to the debate over managed care and the associated pressures for physicians to cut costs to increase profits for private businesses or themselves. For our purposes let us consider that, for a given patient, substituting an alternative treatment for the most effective treatment, for any reason other than patient preference, is a form of rationing, which may involve:

1. Economic rationing - where the most effective treatment for a disease may not be adopted because the cost would outstrip the resources available to society, an institution, or an individual.

2. Rationing to promote public health - where the administration of the most effective treatment for an individual may be outweighed by a detriment to the public health. For example, institutions may limit the widespread use of broad-spectrum antibiotics to prevent the emergence of drug resistant organisms. Rationing to promote public health is a manifestation of the ethical principle of justice.

Many of our standards of care may not only be based on which treatment offers the greatest benefit to the individual patient, but also which treatment can be the most widely distributed to patients with the disease in question, while not consuming undue resources that are also needed for other patients.

If a patient requires oncologic treatment but that treatment involves an exposure risk to other patients and healthcare staff, substituting the standard of care for this one patient in order to mitigate the resultant public health crisis may be justified. If a hospital-wide outbreak were to occur by treating one infected oncology patient, the increased burden of sick patients and healthcare staff on an already stretched medical system might result in otherwise preventable deaths by rendering a shortage of providers and additional necessary resources. Of course, this is completely dependent on the degree of transmission risk and delaying treatment to a cancer patient who is known to be COVID-19 positive is more easily justified than to one of unknown status.

3. Sociopolitical rationing - There are ongoing examples of rationing in our society that conflict with the principle of justice. Namely the unequal distribution of medical care in American society based on class, race, location, employment status and others. This observation is not meant to spark an argument over healthcare reform. Rather, it illustrates an example of the disparity of resources between one population and another.

It could be argued that physicians ration every day. Our time is finite. We spend more of it with some patients than with others. Most of us do not work 365 days each year and we share time with friends and family that could otherwise be spent treating patients. If a patient misses a radiation treatment during the week, many institutions will not make up for it on Saturday. Expedient scheduling for oncologic surgery has always been problematic due to an overload of elective, non-urgent cases. While diminishing the patientcentered clinical ethic, these practices may be necessary to keep institutions financially solvent so they can provide ongoing care.

If resources were truly limitless, withholding the most effective treatment for any reason other than truly informed patient preference would not be ethically sound. Most would agree that healthcare resources are, in fact, finite and that there is a point at which it no longer becomes possible to treat all patients with the means that would provide the greatest chance for the most rapid, maximally effective strategy. As 
our healthcare system becomes ever more dependent on increasingly complex treatments and technology to deliver the most effective care, we inch toward the time when we can no longer afford to provide these treatments to all patients who might warrant them. Unfortunately, the COVID-19 pandemic has propelled rationing from the pages of ethical texts to the forefront of conversation as providers and administrators engage in daily medical decision-making and the formulation of hospital policy in a world of limited resources.

\section{Patient prioritization}

When allocating scarce medical resources to patients, we should minimize death and significant disability. The time-course of illnesses and the degree to which both treatment and its delay will impact survival and quality of life are critical considerations. Rationing decisions that serve public health maximize treatment benefit across society. Considerations such as social or economic status, whether a patient is intrinsically more deserving than another, and the order in which patients present should not have bearing on treatment decisions. [xiii] [xiv] [xv]

Neither COVID-19 patients, nor head and neck cancer patients are intrinsically more deserving of resources than the other. Patients with newly diagnosed head and neck cancer represent an opportunity to treat a disease that carries grave consequences if untreated, but also a high chance of cure (depending on stage) or life extension. Preserving resources for a projected swell of COVID-19 patients prioritizes hypothetical patients for actual ones. Indeed, some emergency capacity should always be maintained to treat life-threatening conditions. Unfortunately, the expected rate of emergency situations in the setting of widespread COVID19 is uncertain, and projections are not always accurate.

\section{The physician-advocate}

Perhaps the disagreement lies not in whether rationing is ethical when resources are scarce, but in the question of who should be responsible for making decisions regarding which patient does or does not receive treatment. Incorporating extrinsic forces into medical decision-making might erode the doctor-patient relationship and lead to injustice. On the other hand, maybe decisions on social justice should not be made by physicians because they are not extensively trained in such matters.

While some physicians may prefer not to make such ethical decisions, it is also likely that many are made uneasy when lawmakers and administrators dictate which treatments are appropriate for specific patients. It is possible that "bedside rationing" by the physician, based on clinical judgement, may be the most effective and practical means of allocating care with the greatest degree of flexibility. In this context, the physician's role in rationing is not only inevitable but has been long established.[xvi]

Physicians, individually and collectively, may feel duty-bound to advocate for their patients to the exclusion of other influences because of the nature of the patient-doctor relationship. Caution in these situations is advisable. Physicians have various degrees of power and influence within an institution. Each patient's access to resources should not depend upon their physician's rank or skill in negotiating for OR time, PPE, ventilators, hospital beds, and ancillary personnel. While these problems have always existed, institutions must recognize and minimize this potential for inequity.

\section{Conclusions}

1. Many head and neck cancer patients in need of surgical excision have an excellent prognosis in the face of a life-threatening disease and this must not be lost amid the crisis of the COVID-19 pandemic. Resources such as COVID-19 testing, operating room time, hospital beds, personnel, and PPE should be granted to these cases whenever available to facilitate standard of care therapy.

2. The risks of COVID-19 to healthcare workers involved in the treatment of head and neck cancer must not be overlooked. Although these risks are still poorly defined, institutions have a duty to protect 
their healthcare workers. Hospital leadership must recognize that staff who routinely participate in longduration, mucosal, aerosol generating cases are at elevated risk and deserve strong consideration for allocation of maximal respiratory PPE. Additional protection should be granted to staff with risk factors for COVID19-related mortality. Pre-operative time-out checklists should incorporate the potential for transmission of infectious disease. When procedures involve aerosolization, this factor and its known risks should be explicitly stated to all staff participating in surgery. Healthcare workers should not be forced to participate in situations that they perceive as unnecessarily risky. Any plan to preserve PPE for potential patients while simultaneously asking healthcare workers to treat curable, life threatening diseases in front of them without full protection should be considered critically.

3. Patients who are to undergo treatment for head and neck cancer should be informed of the elevated risks that COVID-19 presents as well as the uncertainty involved. Patients should undergo pre-treatment COVID-19 testing. For surgery, this should be done as close to the operative date as possible. Patients should also be instructed to self-quarantine for 14 days pre-operatively.

4. Providers must consider their own patients' needs in addition to the public health need in the context of the pandemic. They must weigh a patient's disease severity and acuity as well as the prognosis with treatment. The duty to do what is in the best interest of one patient may at times be at odds with the duty to protect other patients and the medical workforce. A conflict has always existed between our means and methods of distributing care and the core principles of medical ethics. This tension has been underscored by the emergence of COVID-19 and the associated disruption to head and neck oncologic practice. Examples of ways in which benefit to the individual patient has been historically compromised (even if by the smallest degree) are abundant. That is not to say all are clearly unethical and should be discontinued. The current pandemic may provide an opportunity to reassess old habits while navigating new constraints.

\section{References:}

[i]About the NCCN Clinical Practice Guidelines in Oncology https://www.nccn.org/professionals/ default.aspx (as of April 24, 2020)

[ii] Irish Head and Neck Society Considerations on H\&N during COVID-19. From the AHNS COVID-19 Bulletin Board. https://www.ahns.info/wp-content/uploads/2020/03/Irish-Head-and-Neck-Societyconsiderations-on-COVID-20-3-20.pdf March 20, 2020

[iii]Short-Term Recommendations for Non-Small Cell Lung Cancer Management During the COVID-19 Pandemic https://www.nccn.org/covid-19/pdf/COVID_NSCLC.pdf April 13, 2020

[iv] Huang Y, Yang R, Xu Y, Gong P. Clinical characteristics of 36 non-survivors with COVID-19 in Wuhan, China. medRxiv 2020.02.27.20029009; doi:

[v] Zhou F, Yu T, Du R, et al. Clinical course and risk factors for mortality of adult inpatients with COVID-19 in Wuhan, China: a retrospective cohort study. Lancet. 2020.

[vi] Lei S, Jiang F, Su W, et al. Clinical characteristics and outcomes of patients undergoing surgeries during the incubation period of COVID-19 infection. EClinicalMedicine 000 (2020) 100331

[vii] Bhattacharyya N, Fried M. Benchmarks for mortality, morbidity and length of stay for head and neck surgical procedures. Arch Otolaryngol Head Neck Surg. 2001;127(2):127-132

[viii] Nouraei SAR, Middleton SE, Hudovsky A, et al. A national analysis of the outcome of major head and neck cancer surgery: implications for surgeon-level data publication. Clin. Otolaryngol. 2013, 38, 502- 511

[ix] Judson SD, Munster VJ. Nosocomial Transmission of Emerging Viruses via Aerosol-Generating Medical Procedures. Viruses. 2019;11(10).

[x] Tran K, Cimon K, Severn M, Pessoa-Silva CL, Conly J. Aerosol generating procedures and risk of transmission of acute respiratory infections to healthcare workers: a systematic review. PLoS One. 
2012;7(4):e35797.

[xi] Ai T, Yang Z, Hou H, et al. Correlation of Chest CT and RT-PCR Testing in Coronavirus Disease 2019 (COVID-19) in China: A Report of 1014 Cases. Radiology. 2020:200642.

[xii] Malm H, May T, Francis LP, et al. Ethics, Pandemics, and the Duty to Treat. The American Journal of Bioethics. (2008) 8:8, 4-19, DOI: 10.1080/15265160802317974

[xiii] Emanuel E, Persad G, Upshur R, et al. Fair allocation of scarce medical resources in the time of COVID-19. N Engl J Med. 2020 Mar 23. doi: 10.1056/NEJMsb2005114. [Epub ahead of print]

xiv] Leider J, DeBruin D, Reynolds N, et al. Ethical guidance for disaster response, specifically around crisis standards of care: A systematic review. Am J Public Health. 2017 September; 107(9): e1-e9.

[xv] Cao H, Huang S. Principles of scarce medical resource allocation in natural disaster relief: a simulation approach. Med Decis Making. 2012 May-Jun; 32(3):470-6.

[xvi] Ubel PA, Goold S (2001) Recognizing Bedside Rationing. In Weinberg M (Eds.). Medical Ethics: Applying theories and principles to the patient encounter. (p. 488) Amherst, NY: Prometheus Books. 